\title{
Diseño de un sistema de producción de arroz sostenible en Babahoyo, provincia de Los Ríos, Ecuador
}

\section{Design of a sustainable rice production system in Babahoyo, Los Ríos province, Ecuador}

\author{
Oscar Caicedo Camposano ${ }^{1,2}$, Orlando Díaz Romero ${ }^{1}$, Dalton Cadena Piedrahita ${ }^{1,2}$ y Gino Galarza Centeno ${ }^{3}$ \\ Universidad Técnica de Babahoyo ${ }^{1}$ \\ Universidad Nacional Agraria La Molina ${ }^{2}$ \\ Instituto Superior Técnico Eugenio Espejo ${ }^{3}$ \\ *ocamposano@utb.edu.ec
}

DOI: https://doi.org/10.26871/killkana_tecnica.v3i1.472

\begin{abstract}
Resumen
Este trabajo se ajusta para la localidad de Babahoyo, en la provincia de Los Ríos (Ecuador), con el objetivo de diseñar un sistema de producción de arroz que haga sustentable a este monocultivo en Babahoyo y establecer indicadores para la evaluación de la sustentabilidad de este sistema de producción de arroz. Se procederá bajo el siguiente esquema metodológico: con el diseño de los cercos forestales propuestos se podrá establecer un sistema de producción de arroz en donde también se realizaría rotación de cultivos. Los cercos forestales se conformarán por la siembra alternada de inga (Inga edulis Martius), guayacán (Guaiacum sanctum L.) y mango (Manguifera indica), sobre los muros perimetrales de las parcelas de arroz, mientras que la rotación de cultivos será entre arroz (Oryza sativa L.) y soya (Glycine max L.), en el año se cultivará arroz en estación húmeda y soya en estación seca. Los resultados serían la transformación del monocultivo de arroz en un Sistema Sustentable de producción de arroz, lo que permitirá al productor arrocero no depender solo de la producción y comercialización de esta gramínea; con la soya y la inga el sistema permitirá también la fijación de nitrógeno en el suelo y por la naturaleza de sus raíces la soya evitará la compactación del suelo. Asimismo, se espera que el sistema agroforestal propuesto contribuya de un modo eficiente a la captura de carbono. En términos fitosanitarios, la rotación de cultivos interrumpirá los ciclos biológicos de las plagas propias de los cultivares.
\end{abstract}

Palabras clave: Agroforestal, sustentable, arroz, sistema de producción.

\begin{abstract}
This work will be carried out in Babahoyo, in Los Rios province, (Ecuador). The objective is to design a rice production system in order to achieve the monoculture sustainability of this city and to establish the sustainability indicators to evaluate this rice production system. The methodological scheme to be used will be as follows: by designing the proposed forest fences, it will be possible to establish a rice production system in which crop rotation would also take place. The forest fences will be defined by alternating the production of inga (Inga edulis Martius), guayacán (Guaiacum sanctum L.), and mango (Mangifera Indica)on the perimeter walls of rice fields; while there will be a rotation of rice (Oryza sativa L.) and soybean crop (Glycine max L.). Throughout the year, rice will be cultivated during the wet season and soya during the dry one. The transformation of the rice monoculture into a Sustainable System of rice production would be achieved so that the rice farmer will not have to rely only on the production and commercialization of this grass. With soya and inga farming, the system will also allow nitrogen fixation in the soil and, because of the nature of soy roots, soil compaction will be prevented. The proposed agroforestry system is expected to contribute efficiently to carbon capture. In phytosanitary terms, crop rotation will interrupt the biological cycles of cultivar pests.
\end{abstract}

Key words: Agroforestry, sustainable, rice, production system.

\section{INTRODUCCIÓN}

El continente asiático es considerado el mayor productor de arroz a nivel mundial. Cubre el $90.70 \%$ de la producción, seguido de América con el 5,44\%, África con $3.14 \%$, Europa con $0,52 \%$ y Oceanía con $0,20 \%$ (fuente
FAO/ Ecuador en Cifras). En lo que respecta a la comercialización internacional, a nivel de Comunidad Andina de Naciones (CAN), Ecuador es el país que más exporta arroz elaborado, seguido de Perú, mientras que a nivel mundial está entre los 21 países con un porcentaje de $0.31 \%$ ya 
que el primer país exportador de arroz es Tailandia con el $25.63 \%[1]$.

En las importaciones, Filipinas es el país que más importa arroz elaborado a nivel mundial, y Colombia es el mayor importador, dentro de los países que conforman la CAN (Ireta-Paredes et al. 2015). La producción de arroz tiene sus inicios en el Ecuador en el siglo XVIII, pero se fortaleció su consumo y comercialización en el siglo XIX. Este cultivo se desarrolló en un principio en las provincias del Guayas, Los Ríos, Manabí, y Esmeraldas, con el tiempo logró extenderse y comercializarse en la región Sierra. Su fase de industrialización, es decir, la implementación de piladoras (1895) se asentó en Daule, Naranjito y Milagros (Guayas). En términos de comercio internacional, el primer país destino de exportación fue Colombia, y por el lado de las importaciones, en un principio, el consumo de arroz lo se demandaba de Perú [2].

Datos del año 2010 informan, que el Ecuador produjo 1.132.267 toneladas, ya que cuenta con una superficie sembrada de 382.230 hectáreas (ha), de la que se cosecha 363.119 (ha) y rinde 3,12 (Tm/ha), lo que representa una producción nacional del $0.28 \%$ (valor aproximado) a nivel mundial. Se tienen dos tipos de producción en el tiempo: la de invierno y verano, de las cuales se producen arroz con cáscara, sin cáscara, elaborado y quebrado. Si se analiza información desde el año 2001 hasta el 2011, la producción ha tenido de cambios, los que son más visibles en los años 2004 y 2005 debido a problemas de financiamiento, falta de asistencia técnica, elevados costos de producción, etcétera (Sistema de Información del Agro 2017).

Lo que se refiere a la producción por provincias, para el año 2011, el Guayas tuvo un mayor nivel de producción con 120 y 140 quintales por hectárea, los cuales provinieron de los cantones de Daule, Macará y Zapotillo. La producción en los cantones de Pichincha y Portoviejo en Manabí se consideró aceptable por obtener un rendimiento de 100 quintales por hectárea. En Los Ríos, el volumen de producción tuvo para algunos cantones un aumento, mientras que para otros una disminución [3].

La evaluación de la sustentabilidad de sistemas agrarios requiere de la aplicación de nuevos enfoques, tanto analíticos, holísticos y de indicadores. El propósito que persiguen de los científicos que desarrollan y promueven técnicas de manejo sustentables, es llegar a diseñar agroecosistemas con gran resistencia a plagas, buena capacidad de reciclaje y de retención de nutrimentos, así como altos niveles de biodiversidad $[4,5]$.

El objetivo general es proyectar un sistema de producción de arroz sostenible en Babahoyo, provincia de Los Ríos, Ecuador.

\section{MAteriales y Métodos}

Área de Estudio

El establecimiento del sistema de producción de arroz que se propone se realizará en la finca del Ing. Agr. Rolando Jiménez. Esta propiedad tiene una superficie de 12 ha. y está ubicada dentro del Sistema de Riego y Drenaje Babahoyo, en la parroquia Febres Cordero del cantón Babahoyo.

El Sistema de Riego y Drenaje Babahoyo es una irrigación estatal que beneficia a 908 productores arroceros, en donde predomina el monocultivo en una extensión que bordea las 11000 ha en la parroquia antes citada.

Huerta[6] menciona que para que un sistema de producción agrícola sea rentable (tomando en cuenta que el cultivo principal es el arroz), el área para la cual se diseña esta estrategia debe estar en fusión a la siguiente expresión matemática:

$$
S M V=\frac{C F}{\left(R_{L}-R_{R}\right) P}
$$

Donde:

$$
\begin{aligned}
& S M V=\text { Superficie Mínima Viable } \\
& C F=\text { Costo Fijo } \\
& R_{L}=\text { Rendimiento promedio local }(\mathrm{kg} / \mathrm{ha}) \\
& R_{R}=\text { Rendimiento promedio regional }(\mathrm{kg} / \mathrm{ha}) \\
& P=\text { Precio }(\$ / \mathrm{kg})
\end{aligned}
$$

Si bien es cierto, en la Agroforestería no existen modelos o patrones a seguir, el sistema de producción que se propone, se diseñó con fundamento en las exitosas experiencias alcanzadas por científicos agroforestales y agroecólogos como se muestra en las Figuras 1 y 2 . En ese sentido, se diseñó un sistema que contiene dos estrategias agrícolas que pretenden potencializar la sustentabilidad de la producción de arroz; estas son: rotación de cultivos (arroz - leguminosa) y siembra de cercos forestales alrededor de cada una de las parcelas de arroz irrigado, es decir, que básicamente se modificará el monocultivo de arroz (manteniéndose la gramínea como cultivo principal) con un Sistema Agroforestal con las especies Inga edulis Martius, Guaiacum sanctum L. y Manguifera indica (Tommy Atkin), donde se realizará también rotación de cultivos arroz - soya.

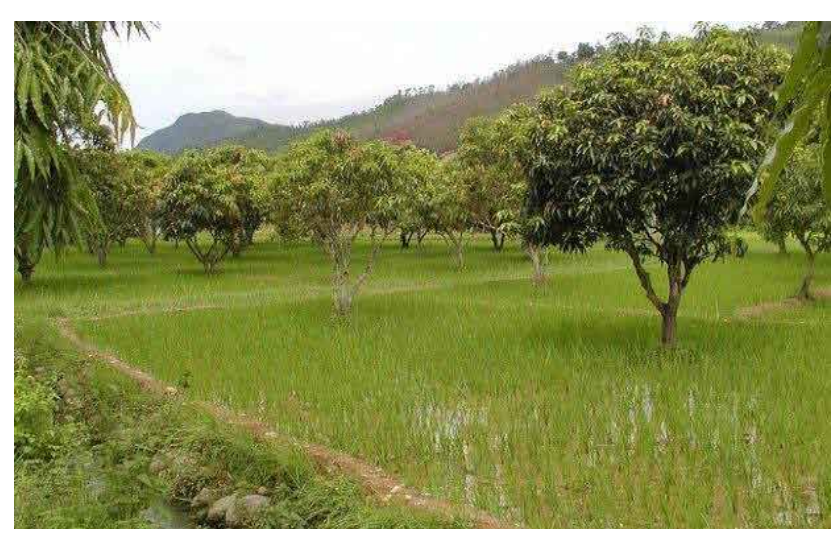

Fig. 1. Sistema Agroforestal (arroz - mago) 


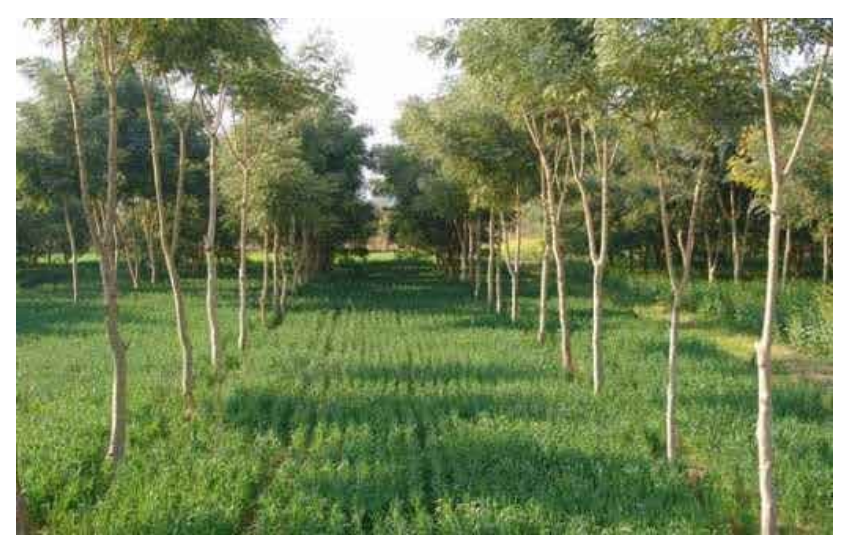

Fig. 2. Sistema Agroforestal (arroz - pachaco (Schizolobium parahyba))

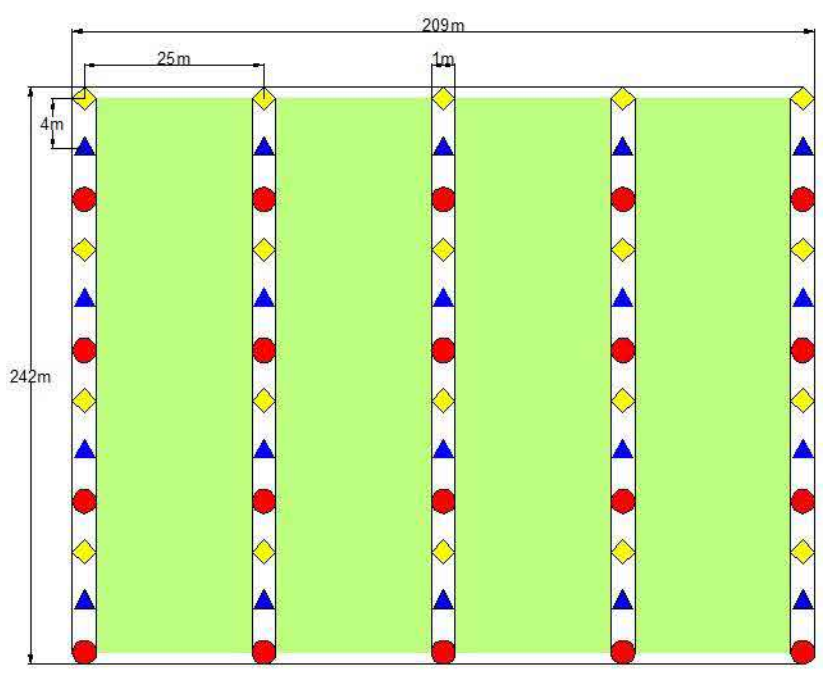

Fig. 3. Diseño del Sistema Agroforestal para producción de arroz con rotación de cultivo arroz - soya

Inga edulis Martius (Guabo)

Guaiacum sanctum L. (Guyacan amarillo)

Manguifera indica (Mango "Tommy Atkin")

Piscinas de arroz con dimensiones de $25 \mathrm{~m}$ x 240m; cercos forestales de las especies antes mencionadas sembrados en hileras a $4 \mathrm{~m}$ de distancia con un patrón de siembra de Guayacán amarillo, Guabo, Mango como se aprecia en la Figura 3. En total se cultivarán cinco ha de rotación arroz - soya (arroz en estación húmeda y soya en estación seca). Se escogió la longitud de $25 \mathrm{~m}$ para facilitar la mecanización agrícola, específicamente en lo relacionado a fumigaciones y cosecha.

La superficie ocupada por los árboles será de 0.163 ha, donde se establecerán por cerco 60 árboles alternados de maderable (guayacán), guabo (leguminosa) y frutal (mango), con lo que se obtiene un total de 540 árboles en la Superficie Mínima Viable.

Con la construcción de indicadores y la evaluación se logra conocer el nivel de sustentabilidad del sistema propuesto, para esto hay que tener en cuenta las tres dimensiones de la sustentabilidad (Ecológica, Socio - Cultural y Económica).
Los parámentos que a considerarse para la evaluación de la sustentabilidad serán los indicadores planteados por Sarandón [7]; subindicadores que fueron ajustados para la realidad de la zona de estudio. Otro aporte importante en este trabajo es el de considerar el secuestro de carbono en el suelo, ya que este es un importante aporte que hacen a la naturaleza los agroecosistemas artificiales.

\section{A. Descripción y ponderación de los indicadores elegidos}

\section{Dimensión Económica}

Para evaluar si el sistema será económicamente viable se eligieron los siguientes indicadores:

A- Autosuficiencia alimentaria. Para este tipo de productores (arroceros), la autosuficiencia alimentaria se consideró fundamental para su sustentabilidad. Esta se estimará, a través de dos indicadores:

A1- Diversificación de la producción. Un sistema es sustentable si la producción alimentaria es diversificada y satisface el nivel nutricional de la familia: (4): más de 5 productos; (3): de 4 a 5; (2): de 3 a 4; (1): de 2 a 3 productos; (0): de 1 a 2 productos.

A2- Superficie de producción de autoconsumo. Un sistema es sustentable si la superficie destinada a la producción de alimentos para el consumo es adecuada con relación a los integrantes del grupo familiar. Variable: superficie de autoconsumo (has)/ integrantes de la familia. (4): más de 1 ha; (3): 1 a 0,5 ha; (2): 0,5-0,3 has; (1): 0,3-0,1 has; (0) $\leq$ 0,1 has.

B-Ingreso neto mensual por grupo. El sistema es sustentable si puede satisfacer las necesidades económicas del grupo familiar. Estos ingresos fueron evaluados en pesos por mes (4): + de 385; (3): 385-285; (2): 285-185; (1): 185100; (0): $<100$.

C- Riesgo económico. Un sistema será sustentable si minimiza el riesgo económico, asegurando la estabilidad en la producción para las generaciones futuras. Se consideraron tres aspectos:

C1- Diversificación para la venta. Un sistema será sustentable si el productor puede comercializar más de 1 producto, ya que si sufriera alguna pérdida o daño del mismo, podría compensarlo con los demás productos que vende. (4): 6 o más productos; (3): 5 a 4 productos; (2): 3 productos; (1): 2 productos; (0): 1 producto.

C2- Número de canales de comercialización. La diversificación comercial disminuye el riesgo económico. (4): 5 o más canales; (3): 4 canales; (2): 3 canales; (1): 2 canales; (0): 1 canal.

C3- Dependencia de insumos externos. Un sistema con una alta dependencia de insumos será insustentable en la línea del tiempo: (4): de 0 a $20 \%$ de insumos externos; (3): de 20 a $40 \%$ de insumos externos; (2): de 40 a $60 \%$ de insumos externos; (1): de 60 a $80 \%$ de insumos externos; (0): de 80 a $100 \%$ de insumos externos.

El valor del indicador económico (IK), se calculó como la suma algebraica de sus componentes multiplicados por su peso o ponderación, de la siguiente manera: 


$$
I K=\frac{\frac{A 1+A 2}{2}+B+\frac{C 1+C 2+C 3}{3}}{3}
$$

\section{Dimensión Ecológica}

Se evaluará a través de tres indicadores:

A-Conservación de la vida de suelo. Un sistema será sustentable si las prácticas mantienen o mejoran la vida en el suelo. Para construir este indicador se tuvieron en cuenta tres subindicadores:

A1- Manejo de la cobertura vegetal. La misma provee al suelo de una protección contra los agentes climáticos y disminuye el riesgo de erosión. (4): $100 \%$ de cobertura; (3): 99 a $75 \%$; (2): 75 a $50 \%$; (1): 50 a $25 \%$; (0): $<25 \%$.

A2- Rotaciones de cultivos. (4) Rota los cultivos todos los años. Deja descansar un año el lote, incorpora leguminosas o abonos verdes; (3): Rota todos los años. No deja descansar el suelo; (2): Rota cada dos o tres años; (1): Realiza rotaciones eventualmente; $(0)$ : No realiza rotaciones.

A3- Diversificación de cultivares en sistema agroforestal con árboles maderables, frutales y fijadores de nitrógeno en el suelo; (4): Diversificación de cultivares en sistema agroforestal con árboles maderables y frutales o maderables y fijadores de nitrógeno en el suelo o frutales y fijadores de nitrógeno en el suelo; (3): Alta diversificación de cultivos, con asociación media entre ellos; (2): Diversificación media, con muy bajo nivel de asociación entre ellos; (1): Poca diversificación de cultivos, sin asociaciones; (0): Monocultivo.

B- Riesgo de erosión. El sistema será sustentable si logra minimizar o evitar la pérdida de suelo debido a la erosión (en este caso, hídrica). Se tuvieron en cuenta tres subindicadores:

B1- Pendiente predominante. (4): del 0 al $5 \%$; (3): del 5 al $15 \%$; (2): del 15 al $30 \%$; (1): del 30 al $45 \%$; (0): mayor al $45 \%$.

B2- Cobertura vegetal. La misma le provee al suelo una protección contra los agentes climáticos y al riesgo de erosión. (4): $100 \%$ de cobertura; (3): 99 a 75\%; (2): 74 a $50 \%$; (1):49 a $25 \%$; (0): $\leq 24 \%$ de cobertura.

C- Manejo de la biodiversidad. La biodiversidad es importante para la regulación del sistema ya que, entre otras funciones, proporciona hábitat y nichos ecológicos para los enemigos naturales. El efecto del sistema de manejo de la finca sobre la biodiversidad, se evaluará a través de dos componentes:

C1- Biodiversidad temporal. Las rotaciones de cultivos en los predios, aumentarán la diversidad en el tiempo. (4): Rota todos los años. Deja descansar un año el potrero o incorpora leguminosas o abonos verdes; (3): Rota todos los años. No deja descansar el suelo; (2): Rota cada 2 o 3 años; (1): Realiza rotaciones eventualmente; (0): No realiza rotaciones.

C2- Biodiversidad espacial. Diversidad de cultivos en el espacio: (4): Establecimiento totalmente diversificado, con asociaciones entre ellos y con vegetación natural; (3): Alta diversificación de cultivos, con media asociación entre ellos; (2): Diversificación media, con muy bajo nivel de asociación entre ellos; (1): Poca diversificación de cultivos, sin asociaciones; (0): Monocultivo.

El indicador o índice que mide el grado de cumplimiento de la dimensión ecológica (IE), se calculó de la siguiente manera:

$$
I E=\frac{\frac{A 1+A 2+A 3}{3}+\frac{2 B 1+B 2}{3}+\frac{C 1+C 2}{2}}{3}
$$

\section{Dimensión Socio-Cultural.}

El grado de satisfacción de los aspectos socioculturales, se evaluará mediante tres indicadores:

A-Satisfacción de las necesidades básicas. Un sistema sustentable es aquel en el cual los agricultores tienen aseguradas sus necesidades básicas. Comprende vivienda, educación, salud, servicios. Se destacan los siguientes subindicadores:

A1- Vivienda. (4): De material terminada. Muy buena. (3): De material terminada. Buena. (2): Regular. Sin terminar o deteriorada. (1): Mala. Sin terminar, deteriorada, piso de tierra; (0): Muy mala.

A2- Acceso a la educación. (4): Acceso a educación superior y/ o cursos de capacitación; (3): Acceso a escuela secundaria; (2): Acceso a la escuela primaria y secundaria con restricciones; (1): Acceso a la escuela primaria; (0): Sin acceso a la educación.

A3- Acceso a salud y cobertura sanitaria: (4): Centro sanitario con médicos permanentes e infraestructura adecuada; (3): Centro sanitario con personal temporario medianamente equipado; (2): Centro sanitario mal equipado y personal temporario; (1): Centro sanitario mal equipado y sin personal idóneo; (0): Sin centro sanitario.

A4- Servicios. (4): Instalación completa de agua, luz y teléfono cercano; (3): Instalación de agua y luz; (2): Instalación de luz y agua de pozo; (1): Sin instalación de luz y agua de pozo cercano; (0): Sin luz y sin fuente de agua cercana.

B- Aceptabilidad del sistema de producción. La satisfacción del productor está directamente relacionada con el grado de aceptación del sistema productivo. (4): Está muy contento con lo que hace. No haría otra actividad aunque esta le reporte más ingresos; (3): Está contento, pero antes le iba mucho mejor; (2): No está del todo satisfecho. Se queda porque es lo único que sabe hacer; (1): Poco satisfecho con esta forma de vida. Anhela vivir en la ciudad y ocuparse de otra actividad; (0): Está desilusionado con la vida que lleva, no lo haría más. Está esperando que se le presente una oportunidad para dejar la producción.

A-Satisfacción de las necesidades básicas. Un sistema sustentable es aquel en el cual los agricultores tienen aseguradas sus necesidades básicas. Comprende vivienda, educación, salud, servicios. Comprende los siguientes subindicadores:

A1- Vivienda. (4): De material terminada. Muy buena. (3): De material terminada. Buena. (2): Regular. Sin termi- 
nar o deteriorada. (1): Mala. Sin terminar, deteriorada, piso de tierra; (0): Muy mala.

A2- Acceso a la educación. (4): Acceso a educación superior y/ o cursos de capacitación; (3): Acceso a escuela secundaria; (2): Acceso a la escuela primaria y secundaria con restricciones; (1): Acceso a la escuela primaria; (0): Sin acceso a la educación.

A3- Acceso a salud y cobertura sanitaria: (4): Centro sanitario con médicos permanentes e infraestructura adecuada; (3): Centro sanitario con personal temporario medianamente equipado; (2): Centro sanitario mal equipado y personal temporario; (1): Centro sanitario mal equipado y sin personal idóneo; (0): Sin centro sanitario.

A4- Servicios. (4): Instalación completa de agua, luz y teléfono cercano; (3): Instalación de agua y luz; (2): Instalación de luz y agua de pozo; (1): Sin instalación de luz y agua de pozo cercano; (0): Sin luz y sin fuente de agua cercana.

B- Aceptabilidad del sistema de producció:. La satisfacción del productor está directamente relacionada con el grado de aceptación del sistema productivo. (4): Está muy contento con lo que hace. No haría otra actividad aunque ésta le reporte más ingresos; (3): Está contento, pero antes le iba mucho mejor; (2): No está del todo satisfecho. Se queda porque es lo único que sabe hacer; (1): Poco satisfecho con esta forma de vida. Anhela vivir en la ciudad y ocuparse de otra actividad; (0): Está desilusionado con la vida que lleva, no lo haría más. Está esperando que se le presente una oportunidad para dejar la producción.

C- Integración social. Se evaluó la relación con otros miembros de la comunidad. (4): Muy alta; (3): Alta; (2): Media; (1): Baja; (0): Nula.

Pero utiliza prácticas de bajos insumos; (0): Sin ningún tipo de conciencia ecológica. Realiza una práctica agresiva al medio por causa de este desconocimiento. Dentro de este objetivo, se consideraron de mayor peso los indicadores de satisfacción de necesidades básicas y el grado de aceptabilidad del sistema productivo:

$$
I S C=\frac{\frac{2(A 1+3 A 2+3 A 3+2 A 4)}{9}}{4}+B+C
$$

\section{B. Índice de sustentabilidad general (ISG)}

Para calcular este índice se adoptó la misma fórmula que propone (Sarandón et al. 2008). Las tres dimensiones reciben una misma valoración porque, en una visión adecuada de la sustentabilidad, estas deben tener la misma importancia y, por lo tanto, el mismo valor. La fórmula propuesta es la que se muestra a continuación:

$$
I S G=\frac{(I K+I E+I S C)}{3}
$$

Siguiendo los criterios de (Sarandón et al. 2008), se puede decir que una finca arrocera con el modelo propuesto sea considerada sustentable, este índice debe ser mayor a 2. Además, ninguna de las tres dimensiones evaluadas debe tener un indicador con un valor menor a 2 .

\section{Indicador "Balance de Reservas de Carbono"}

Lapeyre [8] sostiene que por intercambio gaseoso, las plantas capturan el $\mathrm{CO}_{2}$ de la atmósfera y liberan oxígeno, por lo que en su biomasa (principalmente el follaje) se encuentra gran cantidad de carbono. Luego las hojas de las plantas al cumplir su ciclo natural pasan a formar parte de la materia orgánica del suelo, lugar donde al final es capturado el carbono [9].

El indicador que se menciona es un aporte a los demás indicadores que comúnmente se analizan en la dimensión ambiental de la sustentabilidad; por su complejidad no se le ha considerado dentro de la ecuación de la dimensión antes citada. Sin embargo, es algo que debe ser valorado y cuantificado dentro de los Sistemas Agroforestales con el propósito de conocer la eficiencia de sistemas propuestos (como en este caso) en el proceso de mitigación al cambio climático [10].

En ese sentido se propone monitorear cada cinco años las variables de la ecuación de Reserva de Carbono en el Suelo (RCS), tales como:

Densidad aparente (Da);

Profundidad $(\mathrm{P})$;

Porcentaje de Materia Orgánica (\% MO).

Se propone analizar cada cinco años la reserva del carbono en el suelo, y es entonces que según la diferencia de contenido de carbono entre el análisis de suelo en el año cero y el análisis de suelo en el año cinco, se sabrá el valor certero del secuestro de carbono del suelo en intervalos de cinco años.

Cabe señalar que el porcentaje de MO del suelo será variable en el tiempo y no tendrá un comportamiento lineal, lo mismo sucederá con la $\mathrm{Da}$, es por ello que difícilmente se podría establecer rangos para decir de manera cuantitativa si el sistema es o no sustentable, Por tal motivo, lo que puede plantearse es que si existe captura de carbono en los intervalos de tiempos establecidos, el sistema está siendo sustentable y sobre todo potencializa a la dimensión ecológica.

\section{RESUlTADOS}

El uso de los indicadores propuestos, permitirá detectar, las diferencias entre fincas con monocultivo y la finca con el Sistema de Producción que se plantea en este documento.

El manejo de las finca con el sistema propuesto deberá satisfacer las dimensiones ecológicas, socioculturales y económicas.

Cuando se realice la evaluación de la sustentabilidad, el análisis holístico de los diferentes aspectos de esta permitirá abordar la complejidad del análisis y comprender la relatividad de los resultados según las dimensiones de analizadas.

Transformación del monocultivo de arroz en un Sistema Sustentable de producción de arroz, lo que permitirá al productor arrocero no depender solo de la producción y comercialización de esta gramínea.

Se espera también que el sistema agroforestal propuesto contribuya de forma eficiente a la captura de carbono. 
Con la soya y la inga el sistema permitirá también la fijación de nitrógeno en el suelo y por la naturaleza de sus raíces la soya evitará la compactación del suelo y términos fitosanitarios, de la rotación de cultivos interrumpirá los ciclos biológicos de las plagas propias de los cultivares.

\section{CONClusiones}

En consideración que los arroceros acepten diversificar el monocultivo, esta propuesta podría ser válida en las otras regiones productoras de arroz en Ecuador.

Para una mejor observación de los resultados que se obtengan en la evaluación de la sustentabilidad del modelo propuesto en este documento, puede utilizarse un organizador visual. Para este caso vendría bien usar un gráfico de telaraña, en el cual pueda observarse la dinámica de las variables investigadas.

Este gráfico permitirá detectar los puntos críticos de los sistemas, al dar una idea de la distancia entre la situación ideal y la actual.

La integración de árboles, en el monocultivo de arroz generará una mejor dinámica del manejo, de las sucesiones en sistema de cultivo similar al que se observa en la naturaleza.

\section{REFERENCIAS}

[1] J. Z. Martínez, M. A. Saquet, A. M. Ramírez, R. d. J. L. Zamora, and M. d. L. H. Rodríguez, "Política de estado y producción de cereales: transformaciones territoriales en valles altos de méxico / state policies and cereal production: territorial transformations in high valleys of mexico," Campo - Território: Revista De Geografia Agrária, vol. 12, p. 27, 2018.

[2] G. I. V. Viteri and C. E. Zambrano, "Comercialización de arroz en ecuador: Análisis de la evolución de precios en el eslabón productor-consumidor," Revista Ciencia y Tecnología, vol. 9, no. 2, pp. 11-17, 2016.

[3] Instituto Nacional Autónomo de Investigaciones Agropecuarias, Incremento del nivel productivo, social y económico del sector arrocero ecuatoriano, a través de la inversión pública para la generación y transferencia de tecnología del INIAP.

[4] E. Lichtfouse et al., "Agronomy for sustainable agriculture: a review," in Sustainable agriculture. , Dordrecht, pp. 1-7, 2009.
[5] J. Jacobi, L. Lohse, and J. Milz, "El cultivo de la hoja de coca en sistemas agroforestales dinámicos en los yungas de la paz," Acta Nova, vol. 8, no. 4, pp. 604 630, 2018.

[6] P. Huerta, "Rentabilidad agrícola: Mínima superficie de siembra," 2018.

[7] S. J. Sarandón, M. S. Zuluaga, R. Cieza, L. Janjetic, and E. Negrete, "Evaluación de la sustentabilidad de sistemas agrícolas de fincas en misiones, argentina, mediante el uso de indicadores," Agroecología, vol. 1, pp. 19-28, 2006.

[8] T. Lapeyre, J. Alegre, and L. Arévalo, "Determinación de las reservas de carbono de la biomasa aérea, en diferentes sistemas de uso de la tierra en san martín, perú," Ecología aplicada, vol. 3, no. 1-2, pp. 35-44, 2004.

[9] J. D. Etchevers, V. Saynes, M. Sánchez, and F. D. Roosevelt, Manejo sustentable del suelo para la producción agrícola. El Sistema Agroalimentario de México. Texcoco, México: Editorial del Colegio de Postgraduados, 2016.

[10] J. B. Kauffman, D. C. Donato, and M. F. Adame, Protocolo para la medición, monitoreo y reporte de la estructura, biomasa y reservas de carbono de los manglares (Vol. 117). CIFOR, 2013.

Recibido: 5 de enero de 2019

Aceptado: 25 de marzo de 2019 